ARTICLE

https://doi.org/10.1038/s41467-019-10088-1

\title{
Optimization of 4-1BB antibody for cancer immunotherapy by balancing agonistic strength with FcyR affinity
}

Xinyue $\mathrm{Qi}^{1,2,5}$, Fanlin $\mathrm{Li}^{1,2,5}, \mathrm{Yi} \mathrm{Wu}^{3}$, Chen Cheng ${ }^{1}$, Ping Han ${ }^{1}$, Jieyi Wang ${ }^{3,6}$ \& Xuanming Yang ${ }^{1,2,4,6}$

Costimulation of $T$ cell responses with monoclonal antibody agonists (mAb-AG) targeting 41BB showed robust anti-tumor activity in preclinical models, but their clinical development was hampered by low efficacy (Utomilumab) or severe liver toxicity (Urelumab). Here we show that isotype and intrinsic agonistic strength co-determine the efficacy and toxicity of anti-4-1BB mAb-AG. While intrinsically strong agonistic anti-4-1BB can activate 4-1BB in the absence of FcyRs, weak agonistic antibodies rely on FcyRs to activate 4-1BB. All FcyRs can crosslink anti-41BB antibodies to strengthen co-stimulation, but activating FcyR-induced antibody-dependent cell-mediated cytotoxicity compromises anti-tumor immunity by deleting 4-1BB ${ }^{+}$cells. This suggests balancing agonistic activity with the strength of Fc $\gamma \mathrm{R}$ interaction as a strategy to engineer 4-1BB mAb-AG with optimal therapeutic performance. As a proof of this concept, we have developed LVGN6051, a humanized 4-1BB mAb-AG that shows high anti-tumor efficacy in the absence of liver toxicity in a mouse model of cancer immunotherapy.

\footnotetext{
${ }^{1}$ Sheng Yushou Center of Cell Biology and Immunology, School of Life Sciences and Biotechnology, Shanghai Jiao Tong University, 200240 Shanghai, China. 2 Joint International Research Laboratory of Metabolic \& Developmental Sciences, Shanghai Jiao Tong University, 200240 Shanghai, China. ${ }^{3}$ Lyvgen Biopharma, 201203 Shanghai, China. ${ }^{4}$ Key Laboratory of Systems Biomedicine (Ministry of Education), Shanghai Center for Systems Biomedicine, Shanghai Jiao Tong University, 200240 Shanghai, China. ${ }^{5}$ These authors contributed equally: Xinyue Qi, Fanlin Li. ${ }^{6}$ These authors jointly supervised this work: Jieyi Wang, Xuanming Yang. Correspondence and requests for materials should be addressed to J.W. (email: jieyi.wang@lyvgen.com)

or to X.Y. (email: xuanmingyang@sjtu.edu.cn)
} 
mmune checkpoint blockade antibodies have gained great success in clinic, which aim to release the brake of anti-tumor $\mathrm{T}$ cell response. These treatments are effective for only about $30 \%$ of patients due to various primary or acquired resistance mechanisms in remaining population. Deep sequencing data have revealed that there are multiple mutations in tumor cells, which are important for tumor development from normal cells. At the same time, these mutations have provided numerous 'non-self' targets for immune system recognition, which is the theoretic cornerstone of cancer immunotherapy. Among all the anti-tumor immune responses, $\mathrm{T}$ cell-mediated cytotoxic tumor killing is the key for tumor control. Besides first signal through MHC-peptide-TCR axis, both co-inhibitory and co-stimulatory pathways are critical regulators for $\mathrm{T}$ cell activation ${ }^{1}$. Therefore, agonistic antibodies (Abs) to co-stimulatory molecules and blocking Abs to co-inhibitory molecules are attractive candidates for cancer immunotherapy. While blocking Abs against PD-L1, PD-1, and CTLA-4 have gained great success ${ }^{2}$, clinical development of agonistic Abs against co-stimulation pathways has significantly lagged behind. Currently, there are five immune checkpoint blockade Abs approved for cancer therapy. In contrast, there is no agonistic antibody against co-stimulation receptor approved in clinic.

$4-1 \mathrm{BB}$, as one representative TNF receptor family costimulatory receptor, is expressed on a wide variety of cell types ${ }^{3,4}$, including activated $\mathrm{T}$ cells ${ }^{5}, \mathrm{NK}$ cells $^{6}, \mathrm{DCs}^{7}, \mathrm{~B}$ cells $^{8}$, monocytes $^{9}$, and neutrophils ${ }^{10}$. 4-1BBL-4-1BB interaction can trigger an activation signal in all these cell types. However, anti-41BB-induced $\mathrm{CD}^{+} \mathrm{T}$ responses were thought to play a dominant role in anti-tumor immunity ${ }^{11}$. Anti-4-1BB agonistic Abs could induce more effector molecules released from $\mathrm{CD}^{+} \mathrm{T}$ cells, increase proliferation and decrease apoptosis of $\mathrm{CD} 8^{+} \mathrm{T}$ cells, which all count for the enhanced anti-tumor immunity ${ }^{3,12}$. Despite better or equivalent anti-tumor activity in preclinical models compared with anti-PD-1 and anti-PD-L1 Abs ${ }^{11}$, two anti-4-1BB Abs entered clinical trials, Urelumab and Utomilumab, remained in early stages. These Abs face different challenges in the clinic: while safe, Utomilumab has relatively low efficacy ${ }^{13}$, and Urelumab causes severe liver toxicity despite anti-tumor efficacy ${ }^{14}$. To achieve optimal therapeutic potential, a deeper understanding of the mechanisms behind the anti-tumor and liver toxicity effects of anti-4-1BB Abs is warranted.

The major function of an $\mathrm{Ab}$ is mainly determined by Fab moiety, which contributes to target specificity. However, recently publications have highlighted that the Fc portion also plays important role in regulating $\mathrm{Ab}$ 's function through $\mathrm{Fc}-\mathrm{Fc} \gamma \mathrm{R}$ interaction ${ }^{15-18}$. There are four Fc $\gamma$ Rs (Fc $\gamma$ RI, Fc $\gamma$ RIIB, Fc $\gamma$ RIII, and $F c \gamma R I V)$ in mouse and six Fc $\gamma$ Rs (Fc $\gamma$ RI, Fc $\gamma$ RIIA, Fc $\gamma$ RIIB, Fc $\gamma$ RIIC, Fc $\gamma$ RIIIA, and Fc $\gamma$ RIIIB) in human ${ }^{19}$. Among these $\mathrm{Fc} \gamma \mathrm{Rs}, \mathrm{Fc} \gamma \mathrm{RIIB}$ in both mouse and human is the only inhibitory $\mathrm{Fc} \gamma \mathrm{R}$ to transduce suppressive signal via intracellular ITIM motif. Other $\mathrm{F} c \gamma \mathrm{Rs}$ are activating $\mathrm{Fc} \gamma \mathrm{Rs}$ and transduce activation signals, such as releasing pro-inflammatory cytokines and promoting ADCC effect, through ITAM motif ${ }^{19}$. It has been well known that activating Fc $\gamma$ Rs-mediated ADCC and CDC is required for the efficacy of anti-oncogenic receptor Abs. In this scenario, human IgG1 is commonly chosen to achieve maximum ADCC/ CDC effect, such as Rituximab, Cetuximab, and Herceptin ${ }^{20,21}$. Recent studies have demonstrated other mechanisms of Fc-Fc $\gamma \mathrm{R}$ interaction in regulating the efficacy of immune-modulating Abs. For anti-PD-L1 Abs, mIgG2a or hIgG1 is preferred as activating Fc $\gamma$ R-mediated depletion of PD-L1 ${ }^{+}$immune suppressive cells contributes to its anti-tumor efficacy ${ }^{17,22}$. In contrast, for antiPD-1 Abs, it is better to use mIgG1 or hIgG4 to avoid strong ADCC-mediated depletion of effector $\mathrm{CD}^{+}{ }^{+} \mathrm{T}$ cells ${ }^{17}$. While for anti-CTLA-4 Abs, hIgG1 isotype is critical since depletion of
CTLA- $4^{+}$Treg cells instead of blocking CTLA-4-mediated suppressive signal is dominant mechanism for anti-tumor effect ${ }^{23,24}$ For Abs targeting immune stimulatory molecules, such as antiCD40 Abs, it requires inhibitory Fc $\gamma$ RIIB-mediated crosslinking for agonistic effect ${ }^{15,18,25}$. These complex mechanisms have highlighted the critical role of $\mathrm{Fc}-\mathrm{Fc} \gamma \mathrm{R}$ interaction in modulating therapeutic effect of Abs.

In this study, we have demonstrated, for the first time and to our surprise, that the anti-tumor efficacy and liver toxicity can be separated in natural anti-4-1BB agonist Abs. Further investigations reveal that the intrinsic agonistic strength, isotype, and interaction with $\mathrm{Fc} \gamma \mathrm{Rs}$ are critical for anti-4-1BB antibody activity and liver toxicity. Manipulating these characters will open new direction for 4-1BB and other TNFR family Abs design and optimization.

\section{Results}

Distinct activity and toxicity profiles of anti-4-1BB Abs. To investigate liver toxicity and anti-tumor efficacy of anti-4-1BB Abs in preclinical mouse models, we first screened commercially available rat anti-murine 4-1BB Abs across different tumor types (melanoma B16-OVA and colorectal cancer CT-26) and mouse strains (B6 and Balb/c). In this B16-OVA cell line, OVA is stably expressed and serves as a tumor-specific model antigen. We use B16-OVA model to mimic highly immunogenic cancer in clinic. Both LOB12.3 and 3H3 Abs showed anti-tumor efficacy (Fig. 1a, b) but they exhibited distinct liver toxicity profiles; 3H3 significantly increased alanine transaminase (ALT) levels, whereas LOB12.3 had minimal impact on ALT levels (Fig. 1c, d). We observed similarly increased ALT levels in 3H3-treated naive mice (Fig. 1e, f), suggesting 3H3-induced liver toxicity is independent of tumor burden. Besides elevation of ALT in serum, we also found that immune cell infiltration in the liver of $3 \mathrm{H} 3$ treated mice (Fig. 1g). Our data suggested anti-4-1BB Ab-induced anti-tumor activity and liver toxicity could be separated in natural anti-4-1BB Abs. We, therefore, used LOB12.3 and 3H3 Abs as tool molecules to investigate the underlying mechanism in order to gain insights for clinical development of agonistic anti-4-1BB Abs.

Fc $\gamma$ Rs-crosslinking enhanced the activity of anti-4-1BB Abs. Since $4-1 B B$ is a critical $T$ cell co-stimulatory receptor ${ }^{26}$, we analyzed whether the distinct anti-tumor and liver toxicity profiles of $3 \mathrm{H} 3$ and LOB12.3 were due to differences in their ability to activate $\mathrm{T}$ cells. Using an in vitro co-stimulation assay, we found LOB12.3 and $3 \mathrm{H} 3$ had a comparable ability to activate $\mathrm{T}$ cells in total splenocyte preparations (Fig. 2a). To test whether $\mathrm{T}$ cell activation was dependent on help from other immune cells, we replaced total splenocytes with purified $\mathrm{CD}^{+} \mathrm{T}$ cells in the co-stimulation assay. Surprisingly, only $3 \mathrm{H} 3$ showed a costimulatory effect, suggesting the co-stimulatory ability of $3 \mathrm{H} 3$ does not depend on non-T cells while that of LOB12.3 requires help from other cells (Fig. 2b). This was not due to affinity differences, as affinity to $4-1 \mathrm{BB}$ was comparable (Supplementary Fig. 1A). The IFN- $\gamma$ production was lower from purified $\mathrm{T}$ cells than splenocytes, which may be due to the lack of positive feedback loop between $T$ cells and antigen-presenting cells.

To explore $F c \gamma R$ requirements for co-stimulatory activity of anti-4-1BB Ab, we constructed stable cell lines expressing single mouse Fc $\gamma$ Rs (Supplementary Fig. 2) to mimic Fc $\gamma$ R from non-T cells. In co-cultures of Fc $\gamma$ RIIB-expressing or Fc $\gamma$ RIII-expressing cells and purified $\mathrm{CD} 8^{+} \mathrm{T}$ cells, LOB12.3's co-stimulatory activity was restored (Fig. 2c). This was consistent with the finding that LOB12.3's isotype (rat IgG1) could only bind to mouse FcyRIIB and Fc $\gamma$ RIII (Supplementary Fig. 1B). The co-stimulatory activity 

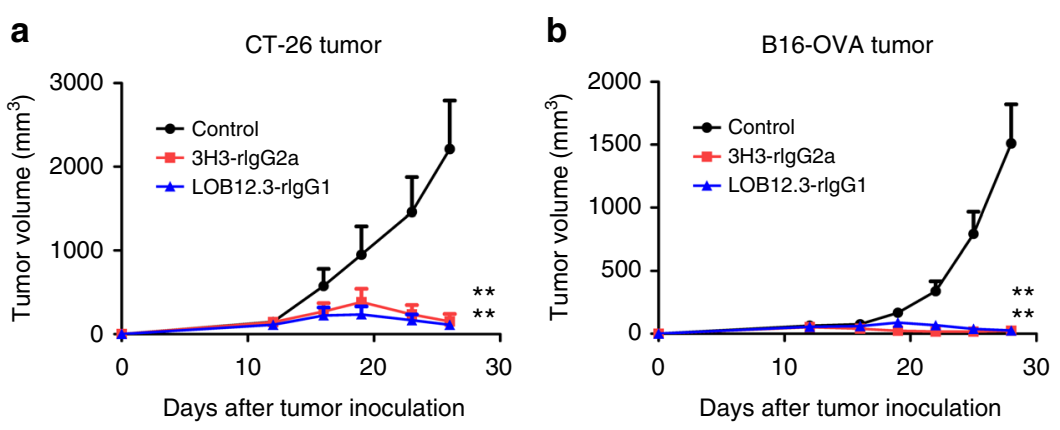

c
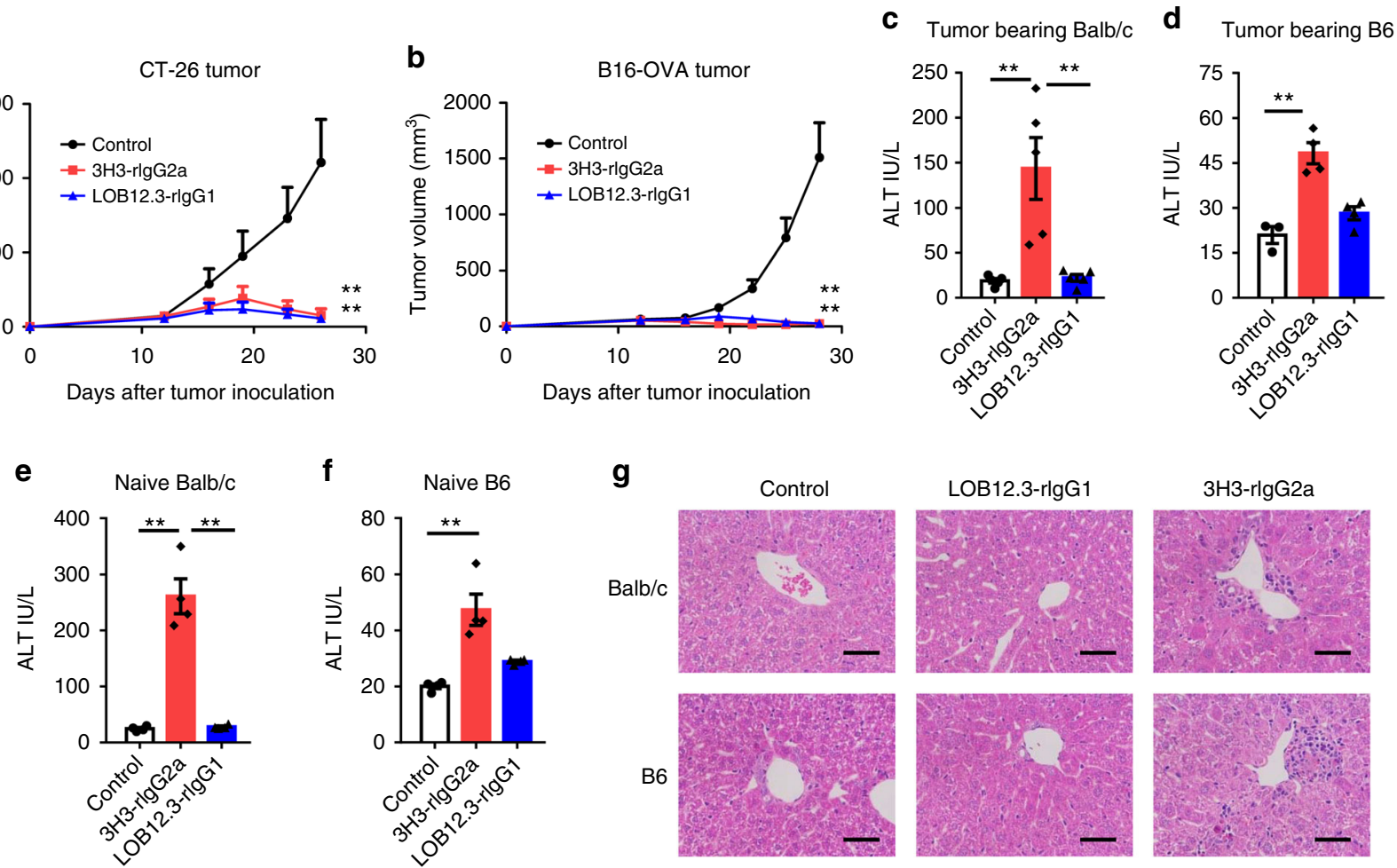

Fig. 1 Distinct activity and toxicity profiles of anti-4-1BB Abs. a WT Balb/c ( $n=4-5 /$ group) or b B6 mice ( $n=5 /$ group) were injected subcutaneously with $1 \times 10^{6}$ (a) CT-26 (b) B16-OVA cells, then $200 \mu$ g of anti-4-1BB (3H3 or LOB12.3) or control IgG was administered on days 9, 16, and 23. Tumor growth was measured twice a week. c, $\mathbf{d}$ Twenty-one days after the first treatment, serum ALT levels in $\mathbf{a}$, $\mathbf{b}$ were measured. e Naive Balb/c ( $n=4 / \mathrm{group}$ ) or $\mathbf{f} B 6$ mice ( $n=4$ /group) were treated with $200 \mu \mathrm{g}$ of anti-4-1BB (3H3 or LOB12.3) or control IgG on days 0 , 7, and 14, and serum ALT levels were measured on day 21. $\mathbf{g}$ Same as in $\mathbf{e}, \mathbf{f}$ liver tissue was stained by HE. Mean \pm SEM are shown. ${ }^{\star} p<0.05,{ }^{\star \star} p<0.01$ compared with control group or as indicated. Scale bar: $50 \mu \mathrm{m}$

of $3 \mathrm{H} 3$ was consistently high whether $\mathrm{Fc} \gamma \mathrm{R}$-expressing cells were present or not although $\mathrm{F} c \gamma \mathrm{R}$-expressing cells enhanced the activity (Fig. 2c). To determine the roles of specific $\mathrm{Fc}$, we engineered chimeric mouse IgG1/IgG2a LOB12.3 and 3H3, while avoiding cross-species immune responses. By using the same in vitro $\mathrm{T}$ cell co-stimulation assay, LOB12.3-mIgG1 showed strong co-stimulation ability when Fc $\gamma$ RIIB or Fc $\gamma$ RIII was present (Fig. 2d). While for LOB12.3-mIgG2a Abs, all Fc $\gamma$ Rs could crosslink and promote its co-stimulation ability (Fig. 2d). When Fc $\gamma$ RIIB or III was present, the agonistic activity of $3 \mathrm{H} 3$ $\mathrm{mIgG1} / \mathrm{mIgG2a}$ was further enhanced (Fig. 2d). These data suggested that agonistic activity of anti-4-1BB Abs varies, which could be further enhanced by FcyRs-mediated crosslinking. Aggregated Abs can perform a crosslinking-like function to promote agonistic effect. To test whether antibody aggregation could contribute to the strong agonistic activity of $3 \mathrm{H} 3$-derived Abs, we performed size-exclusion chromatography (SEC) analysis and there was no antibody aggregation observed from $3 \mathrm{H} 3-$ derived Abs (3H3-rIgG1, 3H3-mIgG1, and 3H3-mIgG2a) (Supplementary Fig. 3). It suggests that the strong agonistic feature of $3 \mathrm{H} 3$-derived Abs is not due to antibody aggregation. Based on these results, we proposed the concept of strong agonistic or weak agonistic anti-4-1BB $\mathrm{Ab}$ : strong agonistic anti-4-1BB $\mathrm{Ab}$ could activate $4-1 \mathrm{BB}$ without the crosslinking from $\mathrm{F} c \gamma \mathrm{R}$ engagement, while weak agonistic anti-4-1BB requires $\mathrm{Fc} \gamma \mathrm{R}$-mediated crosslinking to activate $4-1 \mathrm{BB}$ signaling. Both weak and strong agonistic anti-4-1BB Abs could be further enhanced by Fc $\gamma \mathrm{R}$ mediated crosslinking.

Activating FcyRs compromise anti-4-1BB Abs' activity in vivo. Consistent with potent $\mathrm{T}$ cell activation in vitro, $3 \mathrm{H} 3$-rIgG2a and
LOB12.3-rIgG1 treatment induced strong tumor-specific effector $\mathrm{CD}^{+} \mathrm{T}$ cell responses (Supplementary Fig. 4A). Importantly, $\mathrm{CD}^{+} \mathrm{T}$ cell depletion eliminated the therapeutic effect of anti-4$1 \mathrm{BB}$ Abs, suggesting anti-4-1BB-induced CTL responses are essential for their anti-tumor effect (Supplementary Fig. 4B-E). To test whether anti-4-1BB treatment could generate anti-tumor memory responses, we challenged cured mice with lethal dosage of CT-26 tumor cells. Both 3H3-rIgG2a and LOB12.3rIgG1 showed a protective effect, suggesting an existing tumorspecific memory response (Supplementary Fig. 4F).

Our in vitro data indicated both Fc $\gamma$ RIIB and Fc $\gamma$ RIII can crosslink LOB12.3-rIgG1 to activate 4-1BB co-stimulation pathway on $\mathrm{T}$ cells; we next explored whether FcyRIIB and FcyRIII are required for anti-tumor activity in vivo. In contrast to in vitro observations, anti-tumor efficacy of LOB12.3-rIgG1 was significantly impaired in $\mathrm{Fcgr}_{2} \mathrm{~b}^{-1-}$ but not $\mathrm{Fcgr}^{-1-}$ mice (Fig. 3a, b). Consistent with its strong co-stimulation activity in vitro, 3H3rIgG2a showed anti-tumor activity in both $\mathrm{Fcgr}_{2} \mathrm{~b}^{-/-}$and $\mathrm{Fcgr}^{-/-}$ mice (Fig. 3a, b). To avoid cross-species immune responses, we performed the tumor experiments with $3 \mathrm{H} 3$ and LOB12.3 with mouse IgG1 isotype since mIgG1 has similar mouse Fc $\gamma$ Rs-binding profile as parent rat IgG1 and rat IgG2a. We observed similar phenotype that the anti-tumor activity of LOB12.3-mIgG1 is significantly reduced in $\mathrm{Fcgr} 2 \mathrm{~b}^{-1-}$, while $3 \mathrm{H} 3-\mathrm{mIgG} 1$ showed a weak but significant anti-tumor activity in $\mathrm{Fcgr}_{2} \mathrm{~b}^{-/-}$mice (Fig. 3c). In Fcerlg- ${ }^{-1-}$ and $\mathrm{Fcgr}^{-1-}$ mice, LOB12.3-mIgG1 and 3H3-mIgG1 showed potent anti-tumor activity similar as in WT mice (Fig. 3d, e). These data suggested that mouse immune response to rat isotypes is not the cause in this scenario. Taken together, these data indicate that Fc $\gamma$ RIIB is required for anti-tumor activity of weak agonistic anti-4-1BB Abs in vivo. 


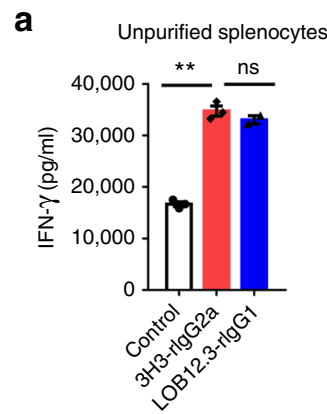

b Purified $C D 8^{+} T$ cells

C

T cells + control cells
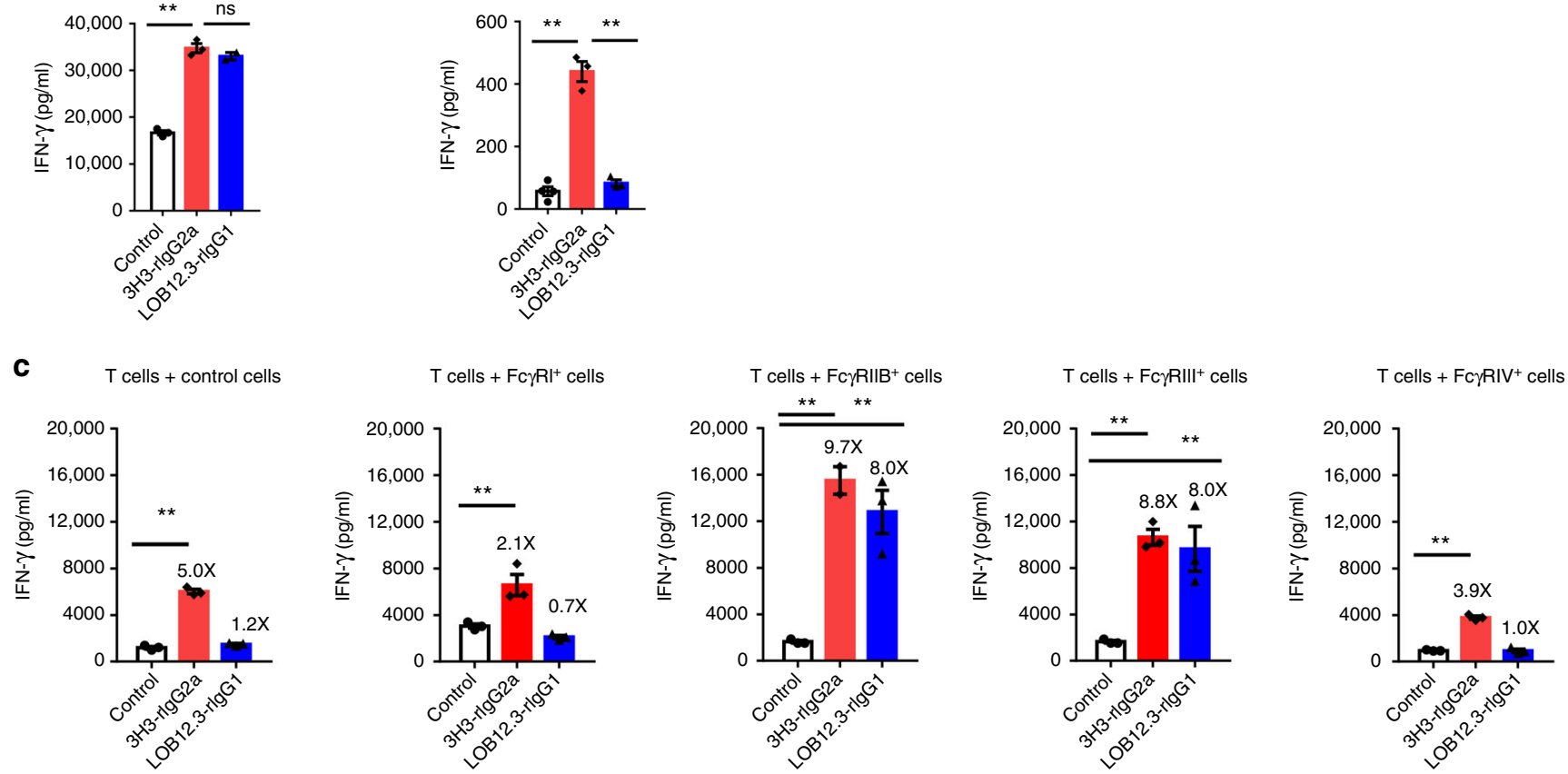

d T cells + control cells
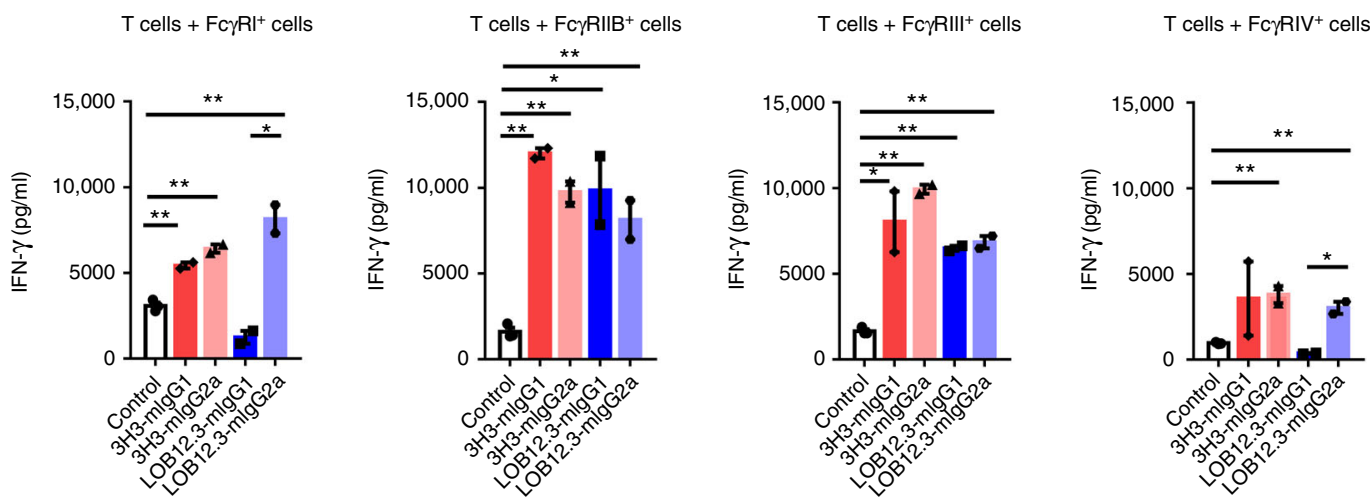

Fig. 2 FcyRs-crosslinking enhanced the activity of anti-4-1BB Abs. a Total splenocytes or $\mathbf{b}$ purified CD8 ${ }^{+}$T cells from B6 mice were stimulated with antiCD3 and indicated anti-4-1BB Abs, 2 days later, the IFN- $\gamma$ in culture medium was analyzed by CBA. c, $\mathbf{d}$ Purified CD8 ${ }^{+}$T cells from B6 mice were stimulated with anti-CD3 and indicated anti-4-1BB Abs when indicated single mouse Fc $\gamma$ R-expressing B16-OVA cells were present, 2 days later, the IFN- $\gamma$ in culture medium was analyzed by CBA. Mean \pm SEM are shown. ${ }^{\star} p<0.05,{ }^{\star \star} p<0.01$ compared with control group or as indicated

Since ADCC is a major event following engagement of activating Fc $\gamma \mathrm{Rs}^{19}$, we hypothesized that downstream signaling of activating Fc $\gamma$ Rs could induce ADCC to delete $4-1 \mathrm{BB}^{+}$effector cells. To test this idea, we compared the anti-tumor activity of LOB12.3-mIgG1, LOB12.3-mIgG2a, 3H3-mIgG1, and 3H3mIgG2a. Mouse IgG2a is similar to human IgG1, which is the strongest isotype to induce ADCC in mice. When strong ADCCinducing isotype $\mathrm{mIgG} 2 \mathrm{a}$ is engineered, the anti-tumor activity is significantly reduced for both $3 \mathrm{H} 3$ and LOB12.3 (Fig. 3f, g). To test whether anti-tumor activity of 3H3-mIgG2a could be restored by blocking Fc $\gamma \mathrm{R}$-mediated ADCC, we generated 3H3mIgG2a with Fc $\gamma$ R-binding null mutations D265A and N297A (DANA), resulting in Abs unable to bind any $\mathrm{Fc}_{\mathrm{Rss}}{ }^{27}$. 3H3mIgG2a-DANA had enhanced anti-tumor activity compared with 3H3-mIgG2a (Fig. 3g), suggesting a negative role for activating Fc $\gamma$ Rs and ADCC in anti-4-1BB-mediated anti-tumor responses. Of note, both LOB12.3-mIgG2a and LOB12.3-mIgG2a-DANA had the same weak anti-tumor effect: for LOB12.3-mIgG2a, the low efficacy is due to strong ADCC-mediated depletion by mIgG2a isotype; while for LOB12.3-mIgG2a-DANA, the lower efficacy is due to lack of Fc $\gamma \mathrm{R}$-mediated crosslinking. 4-1BB ${ }^{+}$ $\mathrm{CD}^{+} \mathrm{T}$ cell numbers were also significantly reduced in $3 \mathrm{H} 3-$ mIgG2a-treated versus 3H3-mIgG1-treated mice, and were restored with 3H3-mIgG2a-DANA treatment (Fig. 3h, Supplementary Fig. 5A), which is consistent with predicted ADCCmediated depletion effect. To provide direct evidence of ADCCinduced $4-1 \mathrm{BB}^{+}$cell depletion, we developed an in vitro assay with target cells $\left(4-1 \mathrm{BB}^{+}\right.$and $\left.4-1 \mathrm{BB}^{-} \mathrm{EL} 4\right)$ and innate effector cells from Rag1 ${ }^{-1-}$ mice. Consistent with in vivo observations, $3 \mathrm{H} 3-\mathrm{mIgG} 2 \mathrm{a}$ induced more killing of $4-1 \mathrm{BB}^{+}$target cells than 3H3-mIgG1, while 3H3-mIgG2a-DANA-induced minimal killing (Fig. 3i, Supplementary Fig. 5B). LOB12.3 Abs showed a similar effect (Fig. 3j). Our data suggest that although activating $F c \gamma R s$ can crosslink and activate 4-1BB, ADCC leads to depletion of $4-1 \mathrm{BB}^{+}$effector cells, compromising anti-tumor activity.

Fab and Fc co-determine liver toxicity by anti-4-1BB Abs. We next analyzed the cell profile in the liver after anti-4-1BB treatment. Infiltration of $\mathrm{CD}_{4} 5^{+}$leukocytes and $\mathrm{CD}^{+}$and $\mathrm{CD}^{+}$ 

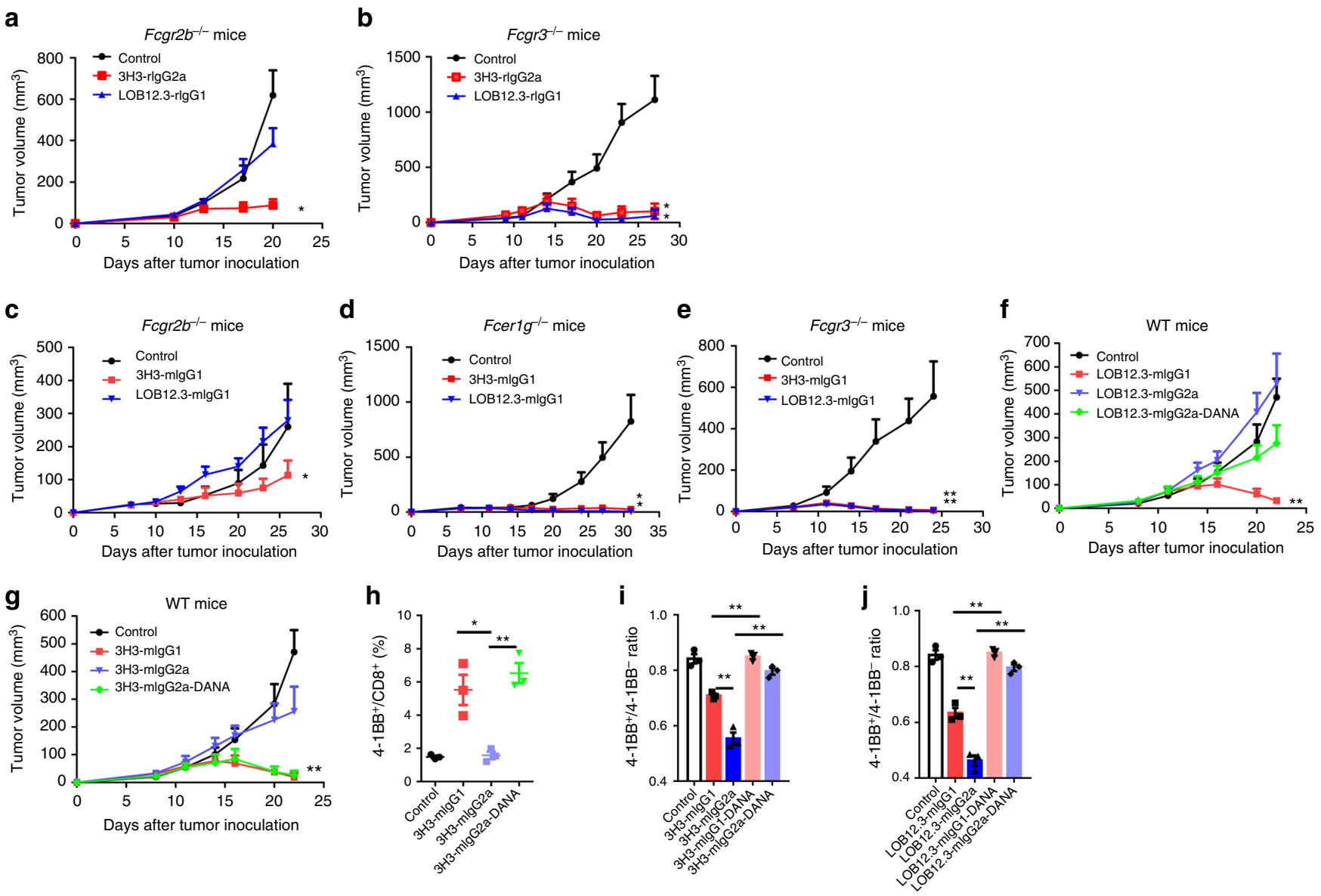

Fig. 3 Activating FcyRs compromise anti-4-1BB Abs' activity in vivo. a Fcgr2b-/- ( $n=6-9 /$ group) and b Fcgr3 ${ }^{-/-}$mice ( $n=5 /$ group) were injected subcutaneously with $1 \times 10^{6}$ B16-OVA cells, then $200 \mu$ g of indicated control lgG or anti-4-1BB Abs were administered on days 9 , 16, and 23 . Tumor growth was measured twice a week. c-e Fcgr2b $b^{-/-}\left(n=6-7 /\right.$ group), Fcerlg ${ }^{-/-}(n=7 /$ group $)$ and Fcgr3 ${ }^{-/-}$mice $(n=5-6 /$ group $)$ were injected subcutaneously with $1 \times 10^{6}$ B16-OVA cells, then $200 \mu \mathrm{g}$ of indicated control lgG or chimeric anti-4-1BB Abs were administered on days 9,16 , and 23 . Tumor growth was measured twice a week. $\mathbf{f}, \mathbf{g}$ WT Balb/c mice ( $n=5 /$ group) were injected subcutaneously with $1 \times 10^{6}$ CT-26 cells, then $200 \mu \mathrm{g}$ of indicated chimeric anti4-1BB Abs or control lgG was administered on days 9, 16, and 23. Tumor growth was measured twice a week. $\mathbf{h}$ Same as in $\mathbf{g}$, splenocytes were analyzed by flow cytometry. $\mathbf{i}$, $\mathbf{j}$ Splenocytes from Rag1-/- mice were co-cultured with EL4-4-1BB + CFSEhigh and EL4-4-1BB- CFSElow cells in the presence of indicated anti-4-1BB Abs. One day later, the ratio of EL4-4-1BB ${ }^{+} / 4-1 B^{-}$was analyzed by flow cytometry. Mean \pm SEM are shown. ${ }^{\star} p<0.05,{ }^{\star *} p<0.01$ compared with control group or as indicated

$\mathrm{T}$ cells dramatically increased in $3 \mathrm{H} 3$-treated, but not in LOB12.3-treated mice (Supplementary Fig. 6A\&B). These CD4 ${ }^{+}$ and $\mathrm{CD}^{+} \mathrm{T}$ cells were activated, with upregulated $\mathrm{CD} 69$ expression and IFN- $\gamma$ production (Fig. 4a, Supplementary Fig. 7). Depletion of $\mathrm{CD}^{+}{ }^{+} \mathrm{T}$ cells decreased ALT while depletion of $\mathrm{CD}^{+} \mathrm{T}$ cells showed no effect (Fig. 4b, Supplementary Fig. 6C), suggesting $\mathrm{CD}^{+} \mathrm{T}$ cell activation is critical for $3 \mathrm{H} 3$-mediated liver toxicity. To explore whether $\mathrm{Fc} \gamma \mathrm{Rs}$ contribute to liver toxicity and potential directions for $\mathrm{Ab}$ optimization, we evaluated 3H3-induced liver damage in $\mathrm{Fcgr}_{2} \mathrm{~b}^{-I-}$ and $\mathrm{Fcgr}^{-1-}$ mice. We found $3 \mathrm{H} 3$-mediated ALT elevation and immune cell infiltration were diminished in $\mathrm{Fcgr}_{2} \mathrm{~b}^{-1-}$ but slightly increased in $\mathrm{Fcgr}^{-/-}$ mice (Fig. 4c, d, Supplementary Fig. 6D\&E). To avoid crossspecies immune responses, we performed the liver toxicity experiments with $3 \mathrm{H} 3$-mouse IgG1 isotype. There is a slightly ALT elevation in 3H3-mIgG1-treated WT mice. However the ALT elevation is significantly increased in $\mathrm{Fcgr}^{-1-}$ and Fcerlg ${ }^{-1-}$ mice, but not in Fcgr2b $b^{-1-}$ mice (Fig. 4e-g). This suggests binding of the $3 \mathrm{H} 3 \mathrm{Fc}$ to inhibitory Fc $\gamma$ RIIB and activating Fc $\gamma \mathrm{RIII}$ has opposing roles in $3 \mathrm{H} 3$-induced liver toxicity, raising the possibility that altering the ratio of activating-to- inhibitory (A/I) Fc $\gamma$ Rs bound to the Fc can modulate liver toxicity effects. We generated 3H3-mIgG1, 3H3-mIgG2a, 3H3-mIgG1DANA, and 3H3-mIgG2a-DANA, which represent isotypes with increasing $\mathrm{A} / \mathrm{I} \mathrm{Fc} \gamma \mathrm{R}$-binding ratio: (mIgG1-DANA or mIgG2aDANA) $<\mathrm{mIgG1}<\mathrm{mIgG} 2 \mathrm{a}$. There is a slightly ALT elevation in 3H3-mIgG1-treated mice, while 3H3-mIgG1-DANA and 3H3mIgG2a-DANA induced significant ALT elevation (Fig. 4h), suggesting an isotype with a low A/I Fc $\gamma$ R-binding ratio is essential for 3H3-induced liver toxicity. We have performed the same experiment with LOB12.3, there is no ALT elevation for LOB12.3-mIgG1, LOB12.3-mIgG1DANA, LOB12.3-mIgG2a, and LOB12.3-mIgG2aDANA (Fig. 4i). Collectively, both a strong agonistic anti-4-1BB Fab and an Fc with a low $\mathrm{A} / \mathrm{I}$ binding ratio are indispensable for anti-4-1BB-mediated liver toxicity.

Activation and toxicity profile of human anti-4-1BB Abs. To test whether the characteristics of strong agonistic and weak agonistic anti-mouse 4-1BB Abs were applicable to anti-human 4$1 \mathrm{BB}$ Abs, we prepared in-house versions of Urelumab (IH-Urelumab) and Utomilumab (IH-Utomilumab). In PBMC and purified $\mathrm{T}$ cell settings, in-house Urelumab was able to co-stimulate 

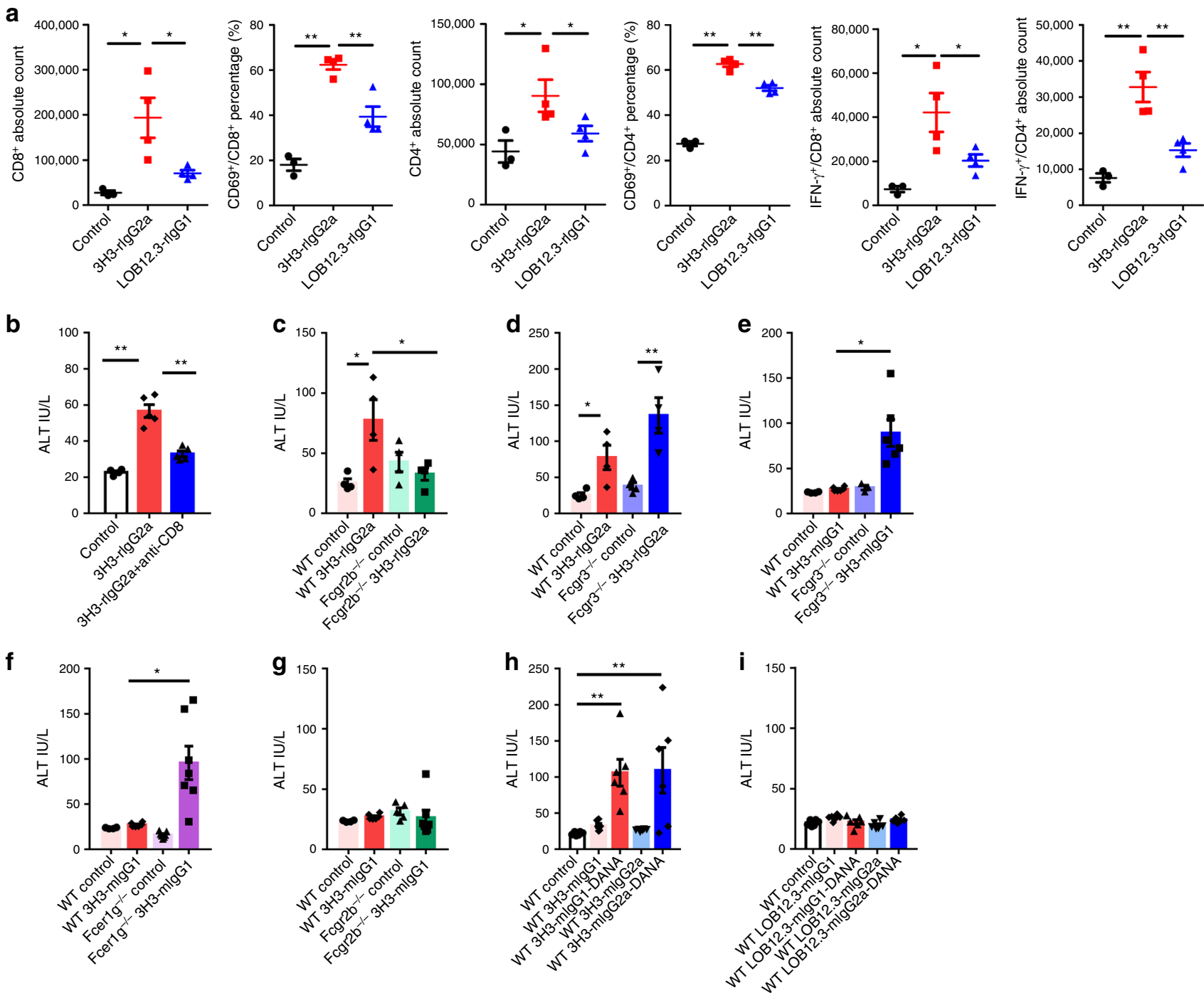

Fig. 4 Fab and Fc co-determine liver toxicity by anti-4-1BB Abs. a After anti-4-1BB Ab treatment, liver leukocytes ( $n=3-4 /$ group) were analyzed by flow cytometry directly or stimulated with PMA/Ionomycin/BFA for $1 \mathrm{~h}$, followed by intracellular staining for IFN- $\gamma$. b WT B6 mice ( $n=4-5 /$ group) were treated with $200 \mu \mathrm{g}$ of anti-4-1BB Abs on days 0, 7, and 14, with $200 \mu \mathrm{g}$ of anti-CD8 administered on days 0 and 7. c, d Fcgr2b-/- ( $n=4 / g r o u p)$ and

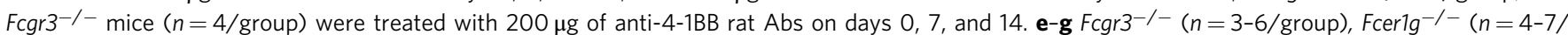
group), and Fcgr $2 b^{-/-}$mice ( $n=4-7 /$ group) were treated with $200 \mu \mathrm{g}$ of anti-4-1BB chimeric Abs on days 0,7 , and 14. h, i WT Balb/c mice ( $n=6 /$ group) were treated with indicated anti-4-1BB chimeric Abs on days 0, 7, and 14. b-i All serum ALT levels were analyzed on day 21 after first antibody treatment. Mean \pm SEM are shown. ${ }^{\star} p<0.05,{ }^{\star \star} p<0.01$ compared with control group or as indicated

$\mathrm{T}$ cells in the absence of Fc $\gamma \mathrm{R}$ (Fig. 5a, b), while in-house Utomilumab required Fc $\gamma$ RIIA-expressing or Fc $\gamma$ RIIB-expressing cells for its agonistic activity (Fig. 5c). This suggests Urelumab is a strong agonistic $\mathrm{Ab}$ and Utomilumab is a weak one. To understand why Urelumab but not Utomilumab induced liver toxicity in clinical studies ${ }^{14}$, we utilized human $4-1 \mathrm{BB}$ transgenic bone marrow chimeric mice to evaluate the liver toxicity of in-house Urelumab and in-house Utomilumab, neither of which recognize murine 4-1BB. Consistent with clinical observations, in-house Urelumab induced significant ALT elevation and immune cell infiltration, whereas in-house Utomilumab did not (Fig. 5d, e). Thus, the MoAs for strong and weak agonistic anti-mouse 4-1BB Abs were also applicable to human anti-4-1BB Abs. Strong agonistic Abs can activate 4-1BB in the absence of $F c \gamma$ Rs with potential to induce liver toxicity, whereas weak agonistic Abs rely on $\mathrm{F} c \gamma \mathrm{R}$ crosslinking for $4-1 \mathrm{BB}$ activation, do not induce liver toxicity, and maintain anti-tumor efficacy.
Engineering potent anti-h4-1BB Ab with limited toxicity. Based on our data with both anti-m4-1BB and anit-h4-1BB Abs, we proposed that a weak agonistic Fab plus an engineered $\mathrm{Fc}$ with selective Fc $\gamma$ RIIB binding might show good efficacy and safety profile. To test our hypothesis, we have engineered a novel antihuman-41BB Ab LVGN6051 to meet these criteria. It is a weak agonistic $\mathrm{Ab}$ which required $\mathrm{F} c \gamma \mathrm{RIIB}$-mediated crosslinking for agonistic activity. In 4-1BB activation reporter assay, LVGN6051 showed significantly improved co-stimulation ability when Fc $\gamma$ RIIB is present (Fig. 6a). Then we compared the T cell co-stimulation ability of LVGN6051, in-house Urelumab, and inhouse Utomilumab. Due to its FcrRIIB selective $\mathrm{Fc}$, LVGN6051 showed T cell co-stimulation ability comparable to in-house Urelumab and superior to in-house Utomilumab when Fc $\gamma$ RIIB-expressing cells are present (Fig. 6b). Furthermore, LVGN6051 showed robust tumor control ability in a wide range of dosage (Fig. 6c, Supplementary Fig. 8A). Most importantly, it 
a

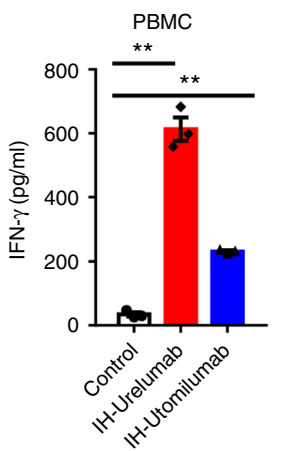

c
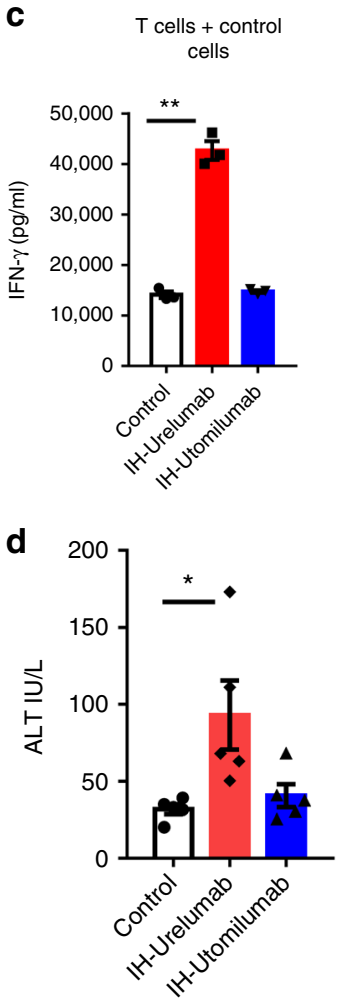

b Purified T cells

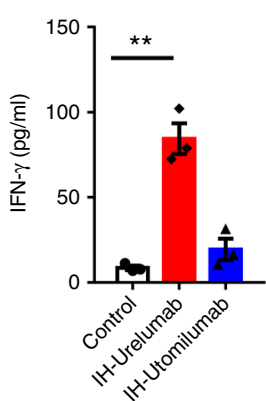

T cells $+\mathrm{hFc}$ RRIA

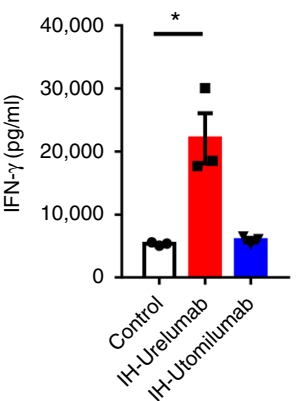

T cells + hFc $\gamma$ RIIA ${ }^{+}$

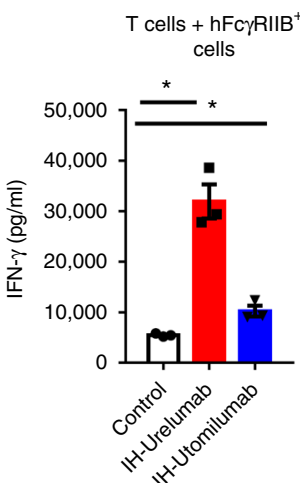

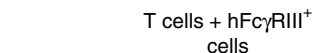
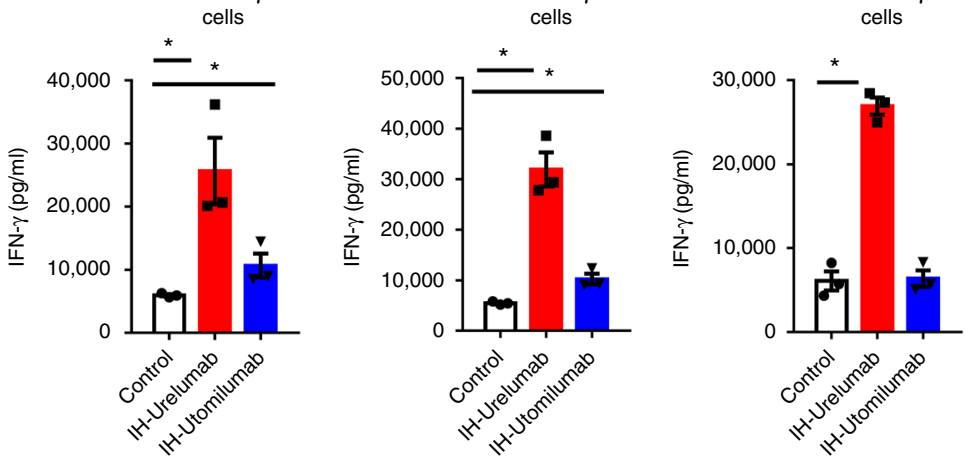

e
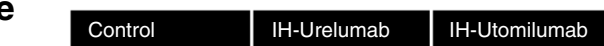

HE
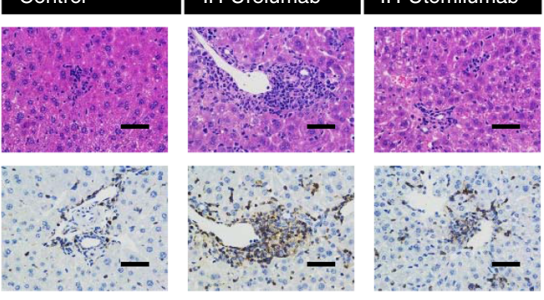

IH-Urelumab

IH-Utomilumab
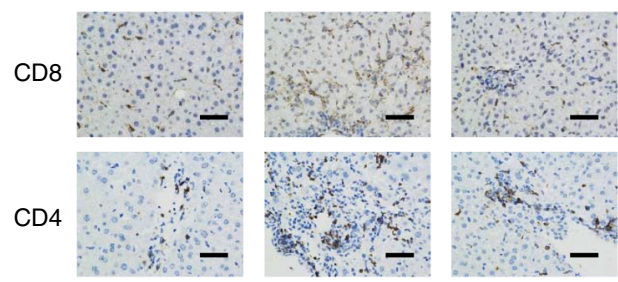

Fig. 5 Activation and toxicity profile of human anti-4-1BB Abs. a Human PBMC or $\mathbf{b}$ purified T cells from human PBMC were stimulated with anti-CD3 and indicated anti-4-1BB Abs. Two days later, the IFN- $\gamma$ in culture medium was analyzed by CBA assay. c Purified T cells from h-4-1BB KI B6 mice were stimulated with anti-CD3 and indicated anti-human 4-1BB Abs in the presence of single human Fc $\gamma$ R-expressing 3 T3 cells. Two days later, the IFN- $\gamma$ in culture medium was analyzed by CBA assay. $\mathbf{d}$ Human 4-1BB KI BMC mice ( $n=5 /$ group) were treated with $200 \mu \mathrm{g}$ of indicated anti-4-1BB Abs on days 0 , 7, and 14. Serum ALT levels were analyzed on day 21. e Same as in d, liver tissue was stained by HE and IHC. Mean \pm SEM are shown. ${ }^{\star} p<0.05,{ }^{\star \star} p<0.01$ compared with control group or as indicated. Scale bar: $50 \mu \mathrm{m}$

did not induce liver toxicity while maintained potent anti-tumor activity (Fig. 6d, Supplementary Fig. 8B). In similar condition, inhouse Urelumab showed potent anti-tumor activity (Fig. 6e). However, it also induced significant liver toxicity (Fig. 6f). This proof-of-concept study demonstrated that our strategy can practically direct the screen and optimization of clinical anti-4$1 \mathrm{BB}$ therapeutic candidates.

\section{Discussion}

Co-stimulation receptor agonistic Abs are important cancer therapeutic candidates. Among them, anti-4-1BB Abs have shown promising anti-tumor effect in preclinical models. However, its clinical development is facing serious challenges due to low efficacy or severe liver toxicity. In this study, we demonstrate for the first time that the anti-tumor efficacy and liver toxicity characteristics of anti-4-1BB agonist Abs can be separated based on the agonistic ability and isotype. The combination of intrinsic agonistic strength and $\mathrm{Fc}$ determine the anti-tumor and liver toxicity property of anti-4-1BB agonist Abs. Anti-4-1BB Fab regions, classified as either strong intrinsic agonistic or weak agonistic Abs, can activate 4-1BB signaling independent of or dependent on $\mathrm{Fc} \gamma \mathrm{R}$ crosslinking, respectively. The contribution of the Fc to anti-4-1BB Ab activity is two-fold. First, all Fc $\gamma$ Rs can crosslink anti-41BB Fc to induce 4-1BB activation. Second, the nature of the $\mathrm{F}_{\mathrm{C}} \gamma \mathrm{R}$ binding the $\mathrm{Fc}$ determines whether ADCC occurs. Activating Fc $\gamma \mathrm{R}$ binding can mediate ADCC, deleting 4$1 \mathrm{BB}^{+}$effector cells modulate liver toxicity and compromising anti-tumor efficacy, while inhibitory Fc $\gamma$ Rs cannot induce ADCC and are purely a crosslinking and activation scaffold (Fig. 7).

Although immune checkpoint blockade Abs have shown promising efficacy in some types of cancer, its overall responding rate 
a

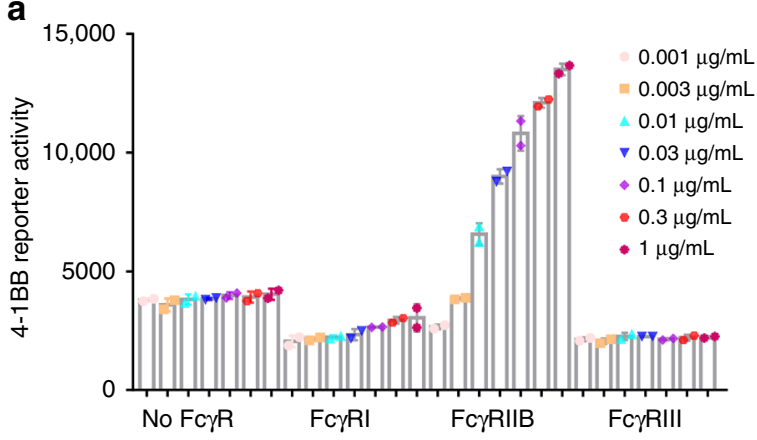

C

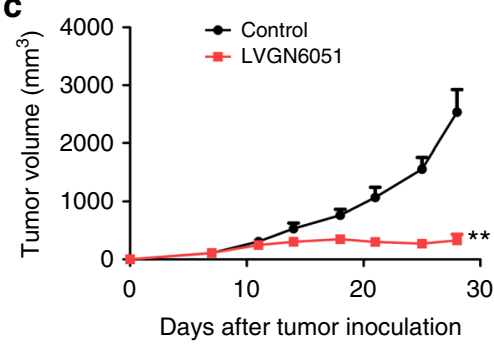

e

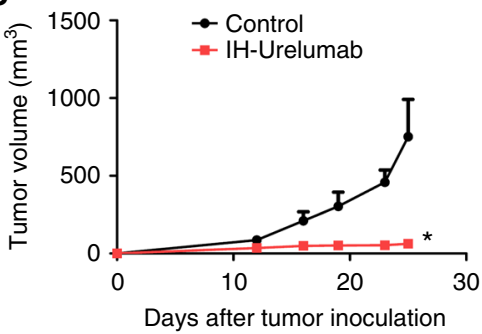

b

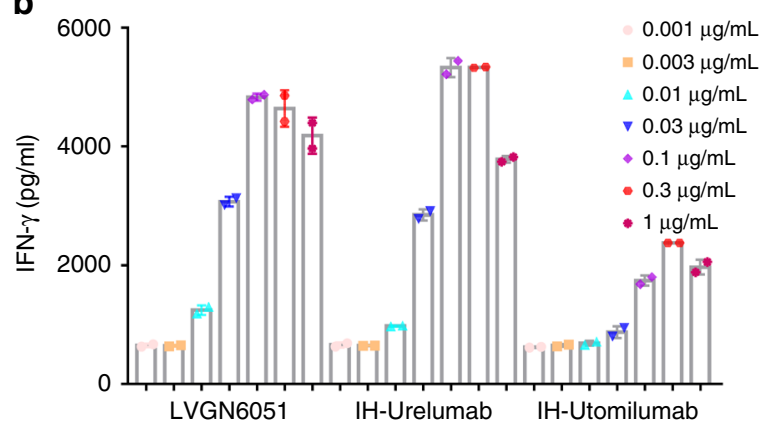

d

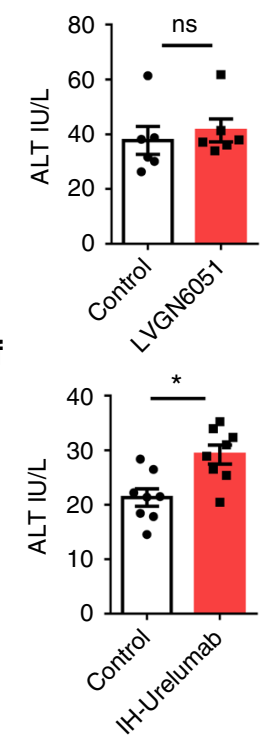

Fig. 6 Engineering potent anti-h4-1BB Ab with limited toxicity. a GS-H2/4-1BB reporter cell line was stimulated with LVGN6051 anti-4-1BB Ab in the presence of single human FcyR-expressing cells. Two days later, reporter activity was analyzed. $\mathbf{b}$ Purified T cells from human PBMC were stimulated with anti-CD3 and indicated anti-4-1BB Abs in the presence of human FcyRIIB-expressing cells. Two days later, the IFN- $\gamma$ in culture medium was analyzed by CBA assay. c Human 4-1BB KI mice ( $n=5-6 /$ group) were treated with $200 \mu \mathrm{g}$ of anti-4-1BB Ab LVGN6051. Tumor growth was measured twice a week. d Same as in c, Serum ALT levels were analyzed on day 21. e Human 4-1BB KI mice ( $n=7 /$ group) were treated with $200 \mu g$ of in-house Urelumab. Tumor growth was measured twice a week. $\mathbf{f}$ Same as in $\mathbf{e}$, Serum ALT levels were analyzed on day 21 after first antibody treatment. Mean \pm SEM are shown. ${ }^{\star} p<$ $0.05,{ }^{* *} p<0.01$ compared with control group or as indicated

is still low, about $30 \% 2,28$. The aim of immune checkpoint blockade is to restore the suppressed anti-tumor immunity to normal level. A lot of intrinsic mechanisms, especially tumor mutation burden which is highlighted by recent publications, have limited the endogenous anti-tumor $\mathrm{T}$ cell responses ${ }^{29}$. Agonist Abs against co-stimulatory receptors in T cells or TNFR in APCs are good choices for enhancing immune response in these scenarios. Despite significant interest and effort on these targets, the fact is that none of them has entered into drug market. One limitation factor is liver toxicity, which is a common side effect for most agonistic TNFR $\mathrm{Abs}^{30-34}$. It largely compromises the safety window as well as anti-tumor efficacy. Based on our data, Fc $\gamma$ RIIB-mediated $\mathrm{CD} 8^{+} \mathrm{T}$ cell activation in the liver contributes to anti-4-1BB-mediated liver toxicity. Since Fc $\gamma$ RIIB is expressed on liver sinusoidal endothelial cells and Kupffer $c^{2}$ ells ${ }^{35}$, we hypothesize that the highly enriched Fc $\gamma$ RIIB environment may provide super crosslinking and activation condition for agonistic anti-4-1BB Abs to induce liver toxicity. It will be interesting to explore whether this can be applied to other TNFR family agonistic Abs and the mechanism of differential anti-4$1 \mathrm{BB} \mathrm{Ab}$ activity in liver and tumor microenvironments. One possible explanation is that the threshold for $\mathrm{T}$ cell activation is different in the liver and tumor. In the liver, most $\mathrm{T}$ cells are naive cells and there is no strong first signal for $\mathrm{T}$ cell activation. Therefore, it requires strong co-stimulation to activate $\mathrm{T}$ cell in the liver, such as strong agonistic anti-4-1BB Ab with low A/I ratio of Fc. In contrast, in the tumor microenvironment, the first signal for $\mathrm{T}$ cell activation is stronger and the requirement for costimulation signal is relatively lower. Therefore, a weak agonistic $\mathrm{Ab}$ with proper crosslinking is sufficient to activate $\mathrm{T}$ cells in the tumor but not strong enough to activate $\mathrm{T}$ cells in the liver.

Based on our observation, we subgroup the agonistic anti-4$1 \mathrm{BB}$ Abs to strong agonistic $\mathrm{Ab}$ and weak agonistic $\mathrm{Ab}$. These two kinds of Abs are not only present in our preclinical research but also exist in clinical usage, indicating the theoretic and practical importance of this definition. The strong agonistic $\mathrm{Abs}(3 \mathrm{H} 3$ and Urelumab) can activate 4-1BB without Fc $\gamma \mathrm{R}$-mediated crosslinking, while the weak agonistic Abs (LOB12.3 and Utomilumab) require $\mathrm{F} c \gamma \mathrm{R}$-mediated crosslinking to activate 4-1BB. The intrinsic mechanism of agonistic strength of different anti-4-1BB Abs remains unclear. Recent research has highlighted the binding epitope is important for the agonistic strength of anti-CD40 $\mathrm{Abs}^{36}$. It will be interesting to investigate whether similar mechanism could be applied to anti-4-1BB Abs. Besides that, the flexibility/rigidity of antibody may contribute to the agonistic strength. Fine crystal structure of antibody-4-1BB complex will 
Strong agonistic anti-4-1BB Abs

$\mathrm{A} / \mathrm{l}$ binding ratio

Activating Fc $\gamma R$ s binding

ADCC \& crosslinking

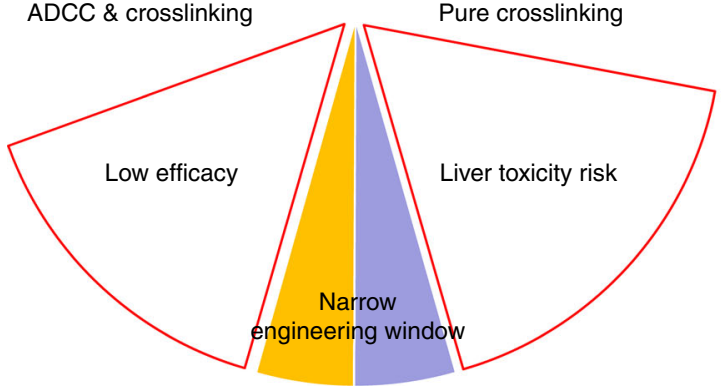

'Sweet point' Fc based strategy
Weak agonistic anti-4-1BB Abs

$\mathrm{A} / \mathrm{I}$ binding ratio

Activating $\mathrm{F}_{\mathrm{c} \gamma \mathrm{R}} \mathrm{s}$ binding

Inhibitory Fc $\gamma \mathrm{R}$ binding

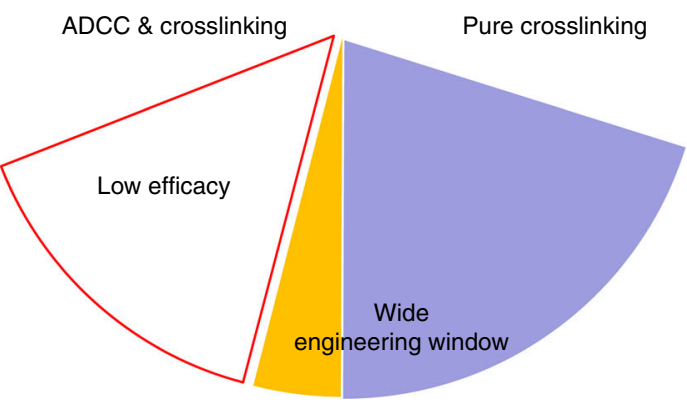

Fc $\gamma$ RIIB-selective Fc based strategy

Fig. 7 Optimization strategies for different anti-4-1BB Abs. Strong agonistic anti-4-1BB Abs can co-stimulate T cell when FcyRs are absent. Weak agonistic anti-4-1BB Abs require FcyR-mediated crosslinking for activity. When anti-4-1BB Abs are crosslinked by activating FcyRs, ADCC will comprise the costimulation ability. When anti-4-1BB Abs are crosslinked by FcyRIIB, it will not cause ADCC and induce strong T cell activation. When strong agonistic anti4-1BB are engineered with low $A / I F c$, it can cause super-activation of $T$ cells and induce liver toxicity. For optimization of strong agonistic anti-4-1BB Abs, it requires fine tuning the $\mathrm{Fc}$ within a narrow range to balancing efficacy and toxicity. For optimization of weak agonistic anti-4-1BB Abs, if equipped with $\mathrm{Fc \gamma RIIB}$ selective $\mathrm{Fc}$, it shows potent anti-tumor activity with desired liver safety profile

provide insights for the underlying mechanism. Previous research has revealed that $\mathrm{F} c \gamma \mathrm{RIIB}$-mediated crosslinking is required for the activity of anti-CD40 and anti-DR5 Abs which are likely to be weak agonistic $\mathrm{Abs}^{15,37}$. Considering the similar activation fashion of TNFR family members, this strong/weak agonistic Ab concept could potentially apply to other TNFR members. It is important to determine the nature of an antibody belongs to strong or weak agonistic $\mathrm{Ab}$ at the first place and then investigate the role of $\mathrm{Fc}$ in regulating the activity of Abs. Furthermore, our findings could open a new direction for TNFR agonistic Ab screening. Most agonistic Abs against TNFR family members are screened through cell line-based assays. If $\mathrm{Fc} \gamma \mathrm{Rs}$ are not present in these assays, the weak agonistic antibody will be excluded. Our results show that the weak agonistic Abs exhibit similar antitumor efficacy as strong agonistic Abs, while their liver toxicity is minimal. Including Fc $\gamma$ Rs in the primary screening assay will provide weak agonistic $\mathrm{Ab}$ candidates for further optimization. Overall, the concept of strong and weak agonistic Abs will greatly clarify the research field of agonistic Abs against TNFR family members and provide new strategy for agonistic Ab screening.

Recent publications have highlighted the important role of $\mathrm{Fc}$ in regulating the activity of cancer therapeutic Abs. Therefore, engineering $\mathrm{Fc}$ to desired $\mathrm{Fc} \gamma \mathrm{R}$-binding profile provides a valuable tool for $\mathrm{Ab}$ optimization. It is reported that the anti-tumor efficacy of anti-CD40 can be further enhanced by engineering the $\mathrm{Fc}$ to have a low $\mathrm{A} / \mathrm{I} \mathrm{Fc} \gamma \mathrm{R}$ binding ratio ${ }^{18}$. Here we propose a modified strategy based on our findings with different categories of anti-4-1BB Abs. For a strong agonistic Fab, an isotype with a low A/I Fc $\gamma$ R-binding ratio can reduce ADCC to improve antitumor activity, but may also cause severe liver toxicity as observed with Urelumab, 2A, or $3 \mathrm{H} 3$ (Fig. 7). The Fc selection for strong agonistic anti-4-1BB Abs needs to be fine titrated to achieve the balance of crosslinking and depletion. For a weak agonistic Fab, an isotype with a low A/I Fc $\gamma$ R-binding ratio (ideally selective binding to $\mathrm{F} c \gamma \mathrm{RIIB}$ ) can have strong anti-tumor activity without liver toxicity (Fig. 7). Thus with proper cataloging, fine-tuning the $\mathrm{A} / \mathrm{I}$-binding ratio through engineering $\mathrm{Fc}$ can provide a powerful tool for anti-4-1BB Ab optimization.

Crosslinking by Fc $\gamma$ RIIB has been shown to play a unique role for the activity of anti-CD40 Abs. Here we present two separated mechanism to explain the role of $\mathrm{Fc}$ in regulating the activity of anti-4-1BB Abs. First, all the Fc $\gamma$ Rs, not limited to Fc $\gamma$ RIIB, could provide crosslinking function for agonistic activity. For example, weak agonistic anti-mouse 4-1BB Abs with mIgG2a isotype could be crosslinked and activated by any mouse $\mathrm{Fc} \gamma \mathrm{R}$-expressing cell lines in vitro (Fig. 2d). While for the same Fabs with mIgG1 isotype, they can only be crosslinked by $\mathrm{mFc} \gamma \mathrm{RIIB} / \mathrm{III}$. Second, if $\mathrm{Fc} \gamma \mathrm{R}$ harbors ITAM motif, the $\mathrm{Fc} \gamma \mathrm{R}^{+}$cells will delete the $4-1 \mathrm{BB}^{+}$ cells through ADCC effect, which will compromise the antitumor activity of anti-4-1BB Abs. If Fc $\gamma$ R harbors ITIM motif, the $\mathrm{Fc} \gamma \mathrm{R}^{+}$cells will only perform a pure crosslinking platform and induce strong activation of 4-1BB signal pathway.

Collectively, the concept of strong agonistic and weak agonistic anti-4-1BB Abs may be applicable to other TNFR family member Abs, or more broadly, receptor agonistic Abs. Proper categorization of these Abs, will further clarify the mechanisms behind therapeutic activity and accelerate clinical agonistic antibody development. Future studies will focus on optimizing anti-h4-1BB agonist Abs for effective tumor control without liver toxicity.

\section{Methods}

Mice. C57BL/6J and Balb/c mice were purchased from Beijing Vital River Laboratory Animal Technology Co., Ltd. (Beijing, China). Fcgr $2 b^{-1-}$ and Fcgr3 ${ }^{-1-}$ mice were purchased from JAX. Human 4-1BB knock-in mice were purchased from Biocytogen, Inc. (Beijing, China). The human 4-1BB extracellular domain is knocked in and replaced with mouse 4-1BB extracellular domain. All mice were maintained under specific pathogen-free conditions. Animal care and use were in accordance with institutional and NIH protocols and guidelines, and all studies were approved by the Animal Care and Use Committee of Shanghai Jiao Tong University.

Cell lines and reagents. B16-OVA was kindly provided by Hans Schreiber (The University of Chicago). Lenti-X 293 was purchased from Clontech. 3T3 and CT-26 were kindly provided by Stem Cell Bank, Chinese Academy of Sciences (Shanghai, China). GS-H2-4-1BB is an engineered reporter cell line stably expressing human 4-1BB receptor whose activation leads to IL-8 production (Genscript). To establish mouse Fc $\gamma$ Rs-expressing B16-OVA cell lines, B16-OVA were infected with mouse $\mathrm{Fc} \gamma \mathrm{R}$-expressing lentivirus. After selection with puromycin, pooled resistant cells were identified by anti-Fc $\gamma \mathrm{R}$ flow cytometry analysis. To establish human $\mathrm{Fc} \gamma \mathrm{R}$ expressing 3T3 cell lines, 3T3 were infected with human Fc $\gamma$ R-expressing lentivirus. After selection with puromycin, pooled resistant cells were identified by antiFc $\gamma$ R flow cytometry. Human PBMCs from cord blood were kindly provided by Shanghai Longyao Biotechnology Co., Ltd. (Shanghai, China). Informed written 
consent was obtained from all study participants and the protocol was approved by the Suqian Obstetrics and Gynecology Hospital Ethics Committee (Jiangsu, China). B16-OVA, Lenti-X 293, 3T3, and their derivatives were cultured in $5 \% \mathrm{CO}_{2}$ and maintained in vitro in DMEM supplemented with $10 \%$ heat-inactivated fetal bovine serum (Gibco), $2 \mathrm{mmol} / \mathrm{L}$ L-glutamine, $100 \mathrm{units} / \mathrm{mL}$ penicillin, and $100 \mu \mathrm{g} /$ $\mathrm{mL}$ streptomycin. Anti-CD8 (YTS 169.4.2) and anti-CD4 (GK1.5) Abs were produced in-house. Anti-mouse 4-1BB (3H3 and LOB12.3) Abs were purchased from BioXcell.

Production of anti-h4-1BB and anti-m4-1BB Abs. For LOB12.3 and 3H3-derived $\mathrm{Abs}$, the $\mathrm{VL}$ and $\mathrm{VH}$ of LOB12.3 and $3 \mathrm{H} 3$ were synthesized according to published amino acid sequence ${ }^{38}$. Using indicated $\mathrm{VH}$ and mouse IgG1 and IgG2a constant region as template, the full length of heavy chain was obtained by overlapping PCR. Using indicated VL and mouse kappa constant region as template, the full length of light chain was obtained by overlapping PCR. These constructs are further cloned into pCDH-EF1-MCS vector. The indicated plasmids containing anti-m41BB were transfected into Lenti-X 293 (Clontech) cells and supernatants were collected and purified by Protein A column according to the manual (Repligen Corporation).

For the anti-h4-1BB Abs Urelumab and Utomilumab, the LC and HC regions of anti-4-1BB were obtained from the published patent US8137667B2 and US 2012/ 0237498A1. The sequences were synthesized by Genewiz (Suzhou, China). The inhouse Urelumab and Utomilumab were produced by ExpiCHO system (Invitrogen) and purified by Protein A column according to the manual (Repligen Corporation).

To generate anti-human 4-1BB antibody LYGN6051, Balb/C and SJL mice were immunized with human 4-1BB protein and pcDNA3.1-human CD137 plasmid. Standard hybridoma method was used to obtain monoclonal Abs against human 4$1 \mathrm{BB}$ using the splenocytes of the immunized mice. The Abs were screened for 4$1 \mathrm{BB}$ binding by standard ELISA and FACS protocols using recombinant 4-1BB protein and $\mathrm{CHO}$ cells transfected to overexpress 4-1BB. High-affinity-binding Abs were then tested in CD8 T cell co-stimulation assays with and without crosslinking by cellular Fc $\gamma$ RIIB. The clone 605 with Fc $\gamma$ RIIB-dependent agonistic activity was selected for further engineering. The heavy and light chain variable region genes were cloned by $5^{\prime}$ RACE system. After humanization using CDR grafting, the variable regions were combined to human kappa and engineered human IgG with point mutations to eliminate binding to the activating $\mathrm{Fc} \gamma \mathrm{Rs}$ including $\mathrm{Fc} \gamma \mathrm{RI}$, Fc $\gamma$ RIIA, and Fc $\gamma$ RIIIA while retain binding to the inhibitory Fc receptor Fc $\gamma$ RIIB and FcRn. The resulting 4-1BB agonist antibody LVGN6051 selectively binds human Fc $\gamma$ RIIB and with no detectable binding to other human-activating Fc $\gamma$ Rs. The recombinant antibody LVGN6051 was produced by stable CHO system and purified by Protein A affinity chromatography followed by ion exchange chromatography. The purified Abs were checked for endotoxin $(<5 \mathrm{EU} / \mathrm{mg})$ and monomerization $(>95 \%)$.

Tumor growth and treatments. Approximately $0.5-1 \times 10^{6}$ B16-OVA or CT- 26 cells were injected subcutaneously on the right flank into 5-12-week-old mice. Tumor volumes were measured along three orthogonal axes $(a, b$, and $c)$ and calculated as tumor volume $=a b c / 2$. After tumor was established $(\sim 9-12$ days), mice were treated with intraperitoneal injections of $200 \mu \mathrm{g}$ anti-4-1BB or control antibody once a week for 3 weeks. For cell-depleting experiments, $200 \mu \mathrm{g}$ of anti$\mathrm{CD} 8$ or anti-CD4 Abs were injected intraperitoneally at the same time as the anti4-1BB treatment.

Measurement of IFN- $\boldsymbol{\gamma}$. OT-I or OT-II peptide-reactive T cells were measured by CBA assay. Spleen or lymph node cells were resuspended in RPMI 1640 supplemented with $10 \% \mathrm{FBS}, 2 \mathrm{mmol} / \mathrm{L}$ L-glutamine, $100 \mathrm{units} / \mathrm{mL}$ penicillin, and $100 \mu \mathrm{g} /$ $\mathrm{mL}$ streptomycin. A total of $1-4 \times 10^{5}$ spleen or lymph node cells were used for the assay. OT-I or OT-II peptide was added at a concentration of $10 \mu \mathrm{g} / \mathrm{mL}$. After $48 \mathrm{~h}$ of incubation, IFN- $\gamma$ production was determined by IFN- $\gamma$ CBA assay (BD Bioscience). GS-H2/4-1BB cells were incubated with $0.001-1 \mu \mathrm{g} / \mathrm{mL}$ of LYGN6051 anti-human 4-1BB Ab in the presence of single human Fc $\gamma \mathrm{R}$-expressing cells. Two days later, IL-8 secretion in the supernatant was determined with human IL-8 HTRF cytokine assay kit (Cisbio) according to the manual.

Detection of endotoxin in mAb preparation. Endotoxin was measured by the limulus amebocyte lysate assay (Cambrex inc., MD). For all $\mathrm{mAb}$ preparations, the amount of endotoxin was determined to be $<0.2$ E.U./mg mAb.

Liver infiltrated leukocytes preparation. Liver tissue was chopped by scissors and digested with $0.2 \mathrm{mg} / \mathrm{mL}$ of Liberase (Roche) and $0.25 \mathrm{mg} / \mathrm{mL}$ of DNase I (Sigma) at $37^{\circ} \mathrm{C}$ for $30 \mathrm{~min}$. The reaction was terminated by adding FBS and EDTA to a final concentration of $10 \%$ and $10 \mathrm{mM}$. The digested suspension was further purified by $70 \%$ and $37 \%$ Percoll gradient centrifugation. The interface layer was collected for further analysis by flow cytometry.

Generation of bone marrow chimeras. WT mice were lethally irradiated with a single dose of $950 \mathrm{rad}$. The next day, irradiated mice were adoptively transferred with $2-3 \times 10^{6}$ h4-1BB KI donor bone marrow cells. Mice were maintained on sulfamethoxazole and trimethoprim (Bactrim) antibiotics diluted in drinking water for 4 weeks after reconstitution. Mice were used for tumor or liver toxicity experiments $\sim 4$ weeks post reconstitution.

Hepatotoxicity measurement. To study the hepatotoxic effects, mice were treated with $200 \mu \mathrm{g}$ of indicated anti-4-1BB Abs i.p. weekly for 3 weeks. Seven days after the last antibody injection, alanine aminotransferase (ALT) levels in the blood were analyzed using the ALT/GPT Enzymatic Assay Kit (BioSino, Beijing, China) following the manufacturer's instructions. Fourteen days after the last antibody injection, liver pathology was assessed by H\&E and IHC staining by Servicebio (Wuhan, China).

In vitro co-stimulation assay. For total mouse splenocyte-based or human PBMC-based co-stimulation assays: unpurified cells $\left(4 \times 10^{5}\right)$ were stimulated with $0.1 \mu \mathrm{g} / \mathrm{mL}$ of anti-CD3 and $2 \mu \mathrm{g} / \mathrm{mL}$ of indicated anti-4-1BB Abs. Two days later, supernatants were collected and IFN- $\gamma$ was measured by CBA. For the purified T cell-based co-stimulation assay, purified T cells $\left(2 \times 10^{5}\right)$ were stimulated with $0.1 \mu \mathrm{g} / \mathrm{mL}$ of anti-CD3 and $2 \mu \mathrm{g} / \mathrm{mL}$ of indicated anti-4-1BB Abs when single Fc $\gamma \mathrm{R}$-expressing cells $\left(2 \times 10^{4}\right)$ are present. Two days later, the supernatants were collected and IFN $-\gamma$ was measured by CBA assay.

Flow cytometric analysis. Single cell suspensions of cells were incubated with anti-CD16/32 (anti-FcrRII/III, clone $2.4 \mathrm{G} 2$ ) for $10 \mathrm{~min}$ and then subsequently stained with conjugated Abs. The purchase and dilution information of all fluorescently labeled monoclonal Abs is listed as the following: $\alpha \mathrm{CD} 4-\mathrm{FITC}$ (\#100406,1:200), aCD8a-AF700 (\#100730, 1:200), aCD69-APC (\#104513, 1:200), aCD44-PE (\#103007, 1:200), aIFN- $\gamma$-APC(\#505810, 1:200), aCD64-APC(\#139305, 1:200), aCD16/32-PE/Cy7(\#101317, 1:200), aCD16.2-FITC(\#149513, 1:200), and a4-1BB-APC( $\# 106109,1: 200)$ from Biolegend; aCD32b-APC(\#17-0321-80, 1:200) from eBioscience. Samples were analyzed on a Cytoflex (Beckman Coulter), and data were analyzed with FlowJo software (TreeStar, Inc.).

Statistical analysis. Mean values were compared using an unpaired Student's twotailed $t$-test. Error bars represent SD or SEM. Statistically significant differences, not significant, $p<0.05$, and $p<0.01$ are noted with ns, ${ }^{\star}$ and ${ }^{* *}$, respectively.

Reporting summary. Further information on research design is available in the Nature Research Reporting Summary linked to this article.

\section{Data availability}

Supporting data of this study are available from the corresponding author on reasonable request. Source data for graphs and tables presented in this manuscript are provided as a Source Data file. LVGN6051 is under clinical trials and its availability may be restricted. LVGN6051 may be available upon request after signing a Material Transfer Agreement with Lyvgen Biopharma.

Received: 1 October 2018 Accepted: 15 April 2019

Published online: 20 May 2019

\section{References}

1. Chen, L. \& Flies, D. B. Molecular mechanisms of T cell co-stimulation and coinhibition. Nat. Rev. Immunol. 13, 227-242 (2013).

2. Zou, W., Wolchok, J. D. \& Chen, L. PD-L1 (B7-H1) and PD-1 pathway blockade for cancer therapy: mechanisms, response biomarkers, and combinations. Sci. Transl. Med. 8, 328rv324 (2016).

3. Watts, T. H. TNF/TNFR family members in costimulation of $\mathrm{T}$ cell responses. Annu. Rev. Immunol. 23, 23-68 (2005).

4. Vinay, D. S. \& Kwon, B. S. 4-1BB signaling beyond T cells. Cell. Mol. Immunol. 8, 281-284 (2011).

5. KwonB. S. \& WeissmanS. M. cDNA sequences of two inducible T-cell genesProc. Natl Acad. Sci. USA 86, 1963-1967 (1989).

6. Melero, I., Johnston, J. V., Shufford, W. W., Mittler, R. S. \& Chen, L. NK1.1 cells express 4-1BB (CDw137) costimulatory molecule and are required for tumor immunity elicited by anti-4-1BB monoclonal antibodies. Cell. Immunol. 190, 167-172 (1998).

7. Wilcox, R. A. et al. Cutting edge: expression of functional CD137 receptor by dendritic cells. J. Immunol. 168, 4262-4267 (2002).

8. Zhang, X. et al. CD137 promotes proliferation and survival of human B cells. J. Immunol. 184, 787-795 (2010).

9. Kienzle, G. \& von Kempis, J. CD137 (ILA/4-1BB), expressed by primary human monocytes, induces monocyte activation and apoptosis of $\mathrm{B}$ lymphocytes. Int. Immunol. 12, 73-82 (2000). 
10. Lee, S. C. et al. Stimulation of the molecule 4-1BB enhances host defense against Listeria monocytogenes infection in mice by inducing rapid infiltration and activation of neutrophils and monocytes. Infect. Immun. 77, 2168-2176 (2009).

11. Melero, I. et al. Monoclonal antibodies against the 4-1BB T-cell activation molecule eradicate established tumors. Nat. Med. 3, 682-685 (1997).

12. Melero, I., Hervas-Stubbs, S., Glennie, M., Pardoll, D. M. \& Chen, L. Immunostimulatory monoclonal antibodies for cancer therapy. Nat. Rev. Cancer 7, 95-106 (2007).

13. Tolcher, A. W. et al. Phase Ib study of utomilumab (PF-05082566), a 4-1BB/ CD137 agonist, in combination with pembrolizumab (MK-3475) in patients with advanced solid tumors. Clin. Cancer Res. 23, 5349-5357 (2017).

14. Segal, N. H. et al. Results from an integrated safety analysis of urelumab, an agonist anti-CD137 monoclonal antibody. Clin. Cancer Res. 23, 1929-1936 (2017).

15. Li, F. \& Ravetch, J. V. Inhibitory Fcgamma receptor engagement drives adjuvant and anti-tumor activities of agonistic CD40 antibodies. Science 333, 1030-1034 (2011).

16. Wilson, N. S. et al. An Fcgamma receptor-dependent mechanism drives antibody-mediated target-receptor signaling in cancer cells. Cancer Cell 19, 101-113 (2011).

17. Dahan, R. et al. FcgammaRs modulate the anti-tumor activity of antibodies targeting the PD-1/PD-L1 axis. Cancer Cell 28, 543 (2015).

18. Dahan, R. et al. Therapeutic activity of agonistic, human anti-CD40 monoclonal antibodies requires selective FcgammaR engagement. Cancer Cell 29, 820-831 (2016).

19. Nimmerjahn, F. \& Ravetch, J. V. Fcgamma receptors as regulators of immune responses. Nat. Rev. Immunol. 8, 34-47 (2008).

20. Clynes, R. A., Towers, T. L., Presta, L. G. \& Ravetch, J. V. Inhibitory Fc receptors modulate in vivo cytotoxicity against tumor targets. Nat. Med. 6, 443-446 (2000).

21. Kurai, J. et al. Antibody-dependent cellular cytotoxicity mediated by cetuximab against lung cancer cell lines. Clin. Cancer Res. 13, 1552-1561 (2007).

22. Zhang, X. et al. Distinct contribution of PD-L1 suppression by spatial expression of PD-L1 on tumor and non-tumor cells. Cell. Mol. Immunol. 16, 392-400 (2018).

23. Du, X. et al. A reappraisal of CTLA-4 checkpoint blockade in cancer immunotherapy. Cell Res. 28, 416-432 (2018).

24. Arce Vargas, F. et al. Fc effector function contributes to the activity of human anti-CTLA-4 antibodies. Cancer Cell 33, 649-663 e644 (2018).

25. White, A. L. et al. Interaction with FcgammaRIIB is critical for the agonistic activity of anti-CD40 monoclonal antibody. J. Immunol. 187, 1754-1763 (2011).

26. Hurtado, J. C., Kim, S. H., Pollok, K. E., Lee, Z. H. \& Kwon, B. S. Potential role of $4-1 \mathrm{BB}$ in $\mathrm{T}$ cell activation. Comparison with the costimulatory molecule CD28. J. Immunol. 155, 3360-3367 (1995).

27. Nimmerjahn, F., Bruhns, P., Horiuchi, K. \& Ravetch, J. V. FcgammaRIV: a novel FcR with distinct IgG subclass specificity. Immunity 23, 41-51 (2005).

28. Baumeister, S. H., Freeman, G. J., Dranoff, G. \& Sharpe, A. H. Coinhibitory pathways in immunotherapy for cancer. Annu. Rev. Immunol. 34, 539-573 (2016).

29. Yarchoan, M., Hopkins, A. \& Jaffee, E. M. Tumor mutational burden and response rate to PD-1 inhibition. New Engl. J. Med. 377, 2500-2501 (2017)

30. Lan, P. et al. TNF superfamily receptor OX40 triggers invariant NKT cell pyroptosis and liver injury. J. Clin. Investig. 127, 2222-2234 (2017).

31. Kimura, K., Nagaki, M., Takai, S., Satake, S. \& Moriwaki, H. Pivotal role of nuclear factor kappaB signaling in anti-CD40-induced liver injury in mice. Hepatology 40, 1180-1189 (2004).

32. Bartkowiak, T. \& Curran, M. A. 4-1BB agonists: multi-potent potentiators of tumor immunity. Front. Oncol. 5, 117 (2015).

33. Bartkowiak, T. et al. Activation of $4-1 \mathrm{BB}$ on liver myeloid cells triggers hepatitis via an interleukin-27-dependent pathway. Clin. Cancer Res. 24, 1138-1151 (2018).
34. Zhang, J. et al. S100A4 blockage alleviates agonistic anti-CD137 antibodyinduced liver pathology without disruption of antitumor immunity. Oncoimmunology 7, e1296996 (2018).

35. Ganesan, L. P. et al. FcgammaRIIb on liver sinusoidal endothelium clears small immune complexes. J. Immunol. 189, 4981-4988 (2012).

36. Yu, X. et al. Complex interplay between epitope specificity and isotype dictates the biological activity of anti-human CD40 antibodies. Cancer Cell 33, 664-675 e664 (2018).

37. LiF. \& RavetchJ. V. Antitumor activities of agonistic anti-TNFR antibodies require differential FcgammaRIIB coengagement in vivo. Proc. Natl Acad. Sci. USA 110, 19501-19506 (2013).

38. Rickert, K. W. et al. Combining phage display with de novo protein sequencing for reverse engineering of monoclonal antibodies. MAbs $\mathbf{8}$, 501-512 (2016).

\section{Acknowledgements}

We thank Dr. Hans Schreiber for providing B16-OVA, Dr. Jie Zhao for comments and editing on this paper. X.Y. was supported by the Program for Professor of Special Appointment (Eastern Scholar) at Shanghai Institutions of Higher Learning (TP2015013), Sheng Yushou Foundation, National Natural Science Foundation of China (81671643), The National Key Research and Development Program of China (2016YFC1303400) and the Recruitment Program of Global Experts (People's Republic of China).

\section{Author contributions}

X.Q. and F.L. conducted most of the experiments. Y.W., C.C. and P.H. provided technical support. X.Y. and J.W. wrote the manuscript. X.Y. and J.W. organized and supervised the project.

\section{Additional information}

Supplementary Information accompanies this paper at https://doi.org/10.1038/s41467 019-10088-1.

Competing interests: The research was funded in part by Lyvgen Biopharma. Y.W. and J.W. are employees of Lyvgen Biopharma. The remaining authors declare no competing interests.

Reprints and permission information is available online at http://npg.nature.com/ reprintsandpermissions/

Journal peer review information: Nature Communications thanks Sergio Quezada and the other, anonymous, reviewer(s) for their contribution to the peer review of this work.

Publisher's note: Springer Nature remains neutral with regard to jurisdictional claims in published maps and institutional affiliations.

Open Access This article is licensed under a Creative Commons Attribution 4.0 International License, which permits use, sharing, adaptation, distribution and reproduction in any medium or format, as long as you give appropriate credit to the original author(s) and the source, provide a link to the Creative Commons license, and indicate if changes were made. The images or other third party material in this article are included in the article's Creative Commons license, unless indicated otherwise in a credit line to the material. If material is not included in the article's Creative Commons license and your intended use is not permitted by statutory regulation or exceeds the permitted use, you will need to obtain permission directly from the copyright holder. To view a copy of this license, visit http://creativecommons.org/ licenses/by/4.0/.

(c) The Author(s) 2019 